TRANSACTIONS OF THE

AMERICAN MATHEMATICAL SOCIETY

Volume 362, Number 8, August 2010, Pages 4229-4242

S 0002-9947(10)05066-X

Article electronically published on March 26, 2010

\title{
ON SOME QUESTIONS RELATED TO THE MAXIMAL OPERATOR ON VARIABLE $L^{p}$ SPACES
}

\author{
ANDREI K. LERNER
}

\begin{abstract}
Let $\mathcal{P}\left(\mathbb{R}^{n}\right)$ be the class of all exponents $p$ for which the HardyLittlewood maximal operator $M$ is bounded on $L^{p(\cdot)}\left(\mathbb{R}^{n}\right)$. A recent result by T. Kopaliani provides a characterization of $\mathcal{P}$ in terms of the Muckenhoupttype condition $A$ under some restrictions on the behavior of $p$ at infinity. We give a different proof of a slightly extended version of this result. Then we characterize a weak type $(p(\cdot), p(\cdot))$ property of $M$ in terms of $A$ for radially decreasing $p$. Finally, we construct an example showing that $p \in \mathcal{P}\left(\mathbb{R}^{n}\right)$ does not imply $p(\cdot)-\alpha \in \mathcal{P}\left(\mathbb{R}^{n}\right)$ for all $\alpha<p_{-}-1$. Similarly, $p \in \mathcal{P}\left(\mathbb{R}^{n}\right)$ does not imply $\alpha p(\cdot) \in \mathcal{P}\left(\mathbb{R}^{n}\right)$ for all $\alpha>1 / p_{-}$.
\end{abstract}

\section{INTRODUCTION}

Let $p: \mathbb{R}^{n} \rightarrow[1, \infty)$ be a measurable function. Denote by $L^{p(\cdot)}\left(\mathbb{R}^{n}\right)$ the space of functions $f$ such that for some $\lambda>0$,

$$
\int_{\mathbb{R}^{n}}|f(x) / \lambda|^{p(x)} d x<\infty
$$

with norm

$$
\|f\|_{L^{p(\cdot)}}=\inf \left\{\lambda>0: \int_{\mathbb{R}^{n}}|f(x) / \lambda|^{p(x)} d x \leq 1\right\} .
$$

Let $\mathcal{P}\left(\mathbb{R}^{n}\right)$ be the class of all functions $p$ for which the Hardy-Littlewood maximal operator $M$ is bounded on $L^{p(\cdot)}\left(\mathbb{R}^{n}\right)$. This class has been a focus of intense study in recent years. By the classical Hardy-Littlewood maximal theorem, any constant function $p \equiv p_{0}$ with $1<p_{0}<\infty$ belongs to $\mathcal{P}\left(\mathbb{R}^{n}\right)$. However, it has been observed quite recently that $\mathcal{P}\left(\mathbb{R}^{n}\right)$ consists of many nontrivial, that is, nonconstant functions. We mention briefly the key known results related to $\mathcal{P}\left(\mathbb{R}^{n}\right)$.

Assume that $p_{-} \equiv \underset{x \in \mathbb{R}^{n}}{\operatorname{essinf}} p(x)>1$ and $p_{+} \equiv \operatorname{ess} \sup p(x)<\infty$. In [5], L. Diening proved that if $p$ satisfies the log-Hölder condition

$$
|p(x)-p(y)| \leq \frac{c}{\log (e+1 /|x-y|)}
$$

and if $p$ is a constant outside some compact set, then $p \in \mathcal{P}\left(\mathbb{R}^{n}\right)$. The second condition on $p$, namely the behavior of $p$ at infinity, was improved independently

Received by the editors June 8, 2008.

2000 Mathematics Subject Classification. Primary 42B25, 46E30.

Key words and phrases. Maximal operator, variable $L^{p}$ spaces.

This work was supported by the Spanish Ministry of Education under the program "Programa Ramón y Cajal, 2006". 
by D. Cruz-Uribe, A. Fiorenza and C. Neugebauer [4, and A. Nekvinda [16]. It was shown in [4] that if $p$ satisfies (1.1) and

$$
\left|p(x)-p_{\infty}\right| \leq \frac{c}{\log (e+|x|)} \quad\left(p_{\infty}>1\right),
$$

then $p \in \mathcal{P}\left(\mathbb{R}^{n}\right)$. In [16, (1.2) is replaced by a slightly more general integral condition. A new approach to these results, as well as an investigation of the limiting cases when $p_{-}=1$ and $p_{+}=\infty$, can be found in the very recent works [2, 3, 7.

Conditions (1.1) and (1.2) are optimal in the pointwise sense; the corresponding examples are contained in [19] and [4. On the other hand, they are not necessary for $p \in \mathcal{P}\left(\mathbb{R}^{n}\right)$. In [17, 18, A. Nekvinda constructed $p \in \mathcal{P}\left(\mathbb{R}^{n}\right)$ satisfying much weaker conditions at infinity than (1.2). In [13, the author established that there exist discontinuous functions $p \in \mathcal{P}\left(\mathbb{R}^{n}\right)$.

In [6, L. Diening showed that $p \in \mathcal{P}\left(\mathbb{R}^{n}\right)$ if and only if there exists $c>0$ such that for any family of pairwise disjoint cubes $\pi$ and any $f \in L^{p(\cdot)}\left(\mathbb{R}^{n}\right)$,

$$
\left\|\sum_{Q \in \pi}\left(|f|_{Q}\right) \chi_{Q}\right\|_{L^{p(\cdot)\left(\mathbb{R}^{n}\right)}} \leq c\|f\|_{L^{p(\cdot)}\left(\mathbb{R}^{n}\right)},
$$

where $f_{Q}=\frac{1}{|Q|} \int_{Q} f$. This result implies, for example, that $p \in \mathcal{P}\left(\mathbb{R}^{n}\right)$ if and only if $p^{\prime} \in \mathcal{P}\left(\mathbb{R}^{n}\right)$, where $p^{\prime}(x)=\frac{p(x)}{p(x)-1}$.

Note that (1.3) with a single cube on the left-hand side would be a full analogue of the classical Muckenhoupt $A_{p}$ condition (cf. 14]) in the context of $L^{p(\cdot)}$ spaces. We give a more precise definition.

Definition 1.1. We say that $p$ satisfies condition $A^{1}(p \in A)$ if $p_{-}>1, p_{+}<\infty$ and there exists $c>0$ such that for any cube $Q$ and any $f \in L^{p(\cdot)}\left(\mathbb{R}^{n}\right)$,

$$
|f|_{Q}\left\|\chi_{Q}\right\|_{L^{p(\cdot)\left(\mathbb{R}^{n}\right)}} \leq c\left\|f \chi_{Q}\right\|_{L^{p(\cdot)\left(\mathbb{R}^{n}\right)}} .
$$

It is natural to ask whether (1.3) can be replaced by $p \in A$. In a recent work [11, T. Kopaliani gave the following partial answer: if $p$ is a constant outside some ball, then $p \in \mathcal{P}\left(\mathbb{R}^{n}\right)$ if and only if $p \in A$. Then this was used in [10] in order to give a new sufficient condition for $p \in \mathcal{P}\left(\mathbb{R}^{n}\right)$ in terms of mean oscillations of $p$.

Observe that the proof in 11] is based essentially on the above mentioned Diening characterization [6], whose proof in turn is long and complicated. In this paper we give a different, self-contained proof of an extended version of Kopaliani's result. Our approach is based on the concept of $A_{\infty}$ weights and on the standard technique which, for example, can be found in the work of B. Jawerth 9 .

Theorem 1.2. Let $p \in A$, and let $E \subset \mathbb{R}^{n}$ be a measurable set of positive finite measure. Then there exists a constant $c>0$ depending on $p, n$ and $E$ such that for any $f \in L^{p(\cdot)}\left(\mathbb{R}^{n}\right)$,

$$
\left\|(M f) \chi_{E}\right\|_{L^{p(\cdot)\left(\mathbb{R}^{n}\right)}} \leq c\|f\|_{L^{p(\cdot)\left(\mathbb{R}^{n}\right)}} .
$$

It is still unclear for us whether the class $\mathcal{P}\left(\mathbb{R}^{n}\right)$ can be fully characterized in terms of condition $A$. However, our next result shows that the weak type $(p(\cdot), p(\cdot))$ property of $M$ is equivalent to $p \in A$ for radially decreasing $p$.

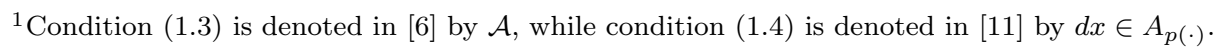
We prefer to denote (1.4) by $A$, and we hope that this will not mislead the reader.
} 
Given a function $p$, we say that $M$ is of weak type $(p(\cdot), p(\cdot))$ if there exists $c>0$ such that for any $f \in L^{p(\cdot)}\left(\mathbb{R}^{n}\right)$,

$$
\sup _{\alpha>0} \alpha\left\|\chi_{\{x:|M f(x)|>\alpha\}}\right\|_{L^{p(\cdot)\left(\mathbb{R}^{n}\right)}} \leq c\|f\|_{L^{p(\cdot)}\left(\mathbb{R}^{n}\right)} .
$$

It is easy to see that the weak type $(p(\cdot), p(\cdot))$ property of $M$ implies $p \in A$. Using Theorem 1.2, we obtain that the converse is also true for radially decreasing $p$. Recall that a function $p$ is radially decreasing if $p(x)=\rho(|x|)$, where $\rho$ is a nonincreasing function on $(0, \infty)$. The following theorem can be viewed as an analogue of Muckehnoupt's characterization [14] of the weighted weak $L^{r}$ boundedness of $M$ in terms of the $A_{r}$ condition.

Theorem 1.3. Let $p$ be a radially decreasing function with $p_{-}>1$ and $p_{+}<\infty$. Then $M$ is of weak type $(p(\cdot), p(\cdot))$ if and only if $p \in A$.

Our next result is closely related to the author's work [13]. It was shown there that any pointwise multiplier for $B M O\left(\mathbb{R}^{n}\right)$ generates a function $p \in \mathcal{P}\left(\mathbb{R}^{n}\right)$. A function $g$ is called a pointwise multiplier for $B M O$ if $f g \in B M O$ for any $f \in B M O$. The main result of [13] states the following.

Theorem A. If $p$ is a pointwise multiplier for $B M O\left(\mathbb{R}^{n}\right)$ with $p_{-}>0$, then there exists a constant $\alpha>0$ such that $p(\cdot)+\alpha \in \mathcal{P}\left(\mathbb{R}^{n}\right)$.

Observe that conditions (1.1) and (1.2) imply that $p$ is a pointwise multiplier for $B M O\left(\mathbb{R}^{n}\right)$. Therefore, the following was asked in [13] .

Question 1.4. Does any pointwise multiplier for $B M O$ with $p_{-}>1$ belong to $\mathcal{P}\left(\mathbb{R}^{n}\right)$ ?

Taking into account Theorem A, this question naturally leads to the following one, which is also of some independent interest.

Question 1.5. Let $p \in \mathcal{P}\left(\mathbb{R}^{n}\right)$. Does this imply $p(\cdot)-\alpha \in \mathcal{P}\left(\mathbb{R}^{n}\right)$ for any $\alpha<$ $p_{-}-1$ ?

The following question, similar to Question 1.5, was asked in 8$]$.

Question 1.6. Let $p \in \mathcal{P}\left(\mathbb{R}^{n}\right)$. Does this imply $\alpha p(\cdot) \in \mathcal{P}\left(\mathbb{R}^{n}\right)$ for any $\alpha>1 / p_{-}$?

Our next result shows that the answers to all the above questions are negative.

Theorem 1.7. Let $n=1$. Also, let $q>1$ and $\delta>0$. There exists a nonnegative function $p_{0}$ satisfying the following properties:

(i) $p_{0}$ is a pointwise multiplier for $B M O(\mathbb{R})$;

(ii) if $q(q-1) \leq \delta$, then $p_{q, \delta}(x)=q+\delta p_{0}(x) \notin A$.

It follows immediately from this theorem that for any $q>1$ and $\delta>0$ such that $q(q-1) \leq \delta$, the function $p_{q, \delta}$ yields a counterexample to Question 1.4. This, along with Theorem A, gives a counterexample to Question 1.5. Finally, applying Theorem A to $p_{0}(\cdot)+\varepsilon_{0}$, where $\varepsilon_{0}>0$, we get that there exists $\alpha_{0}>0$ such that $p_{0}(\cdot)+\alpha_{0} \in \mathcal{P}\left(\mathbb{R}^{n}\right)$. Taking this function and $\alpha>1 / \alpha_{0}$ such that $\alpha_{0}\left(\alpha \alpha_{0}-1\right) \leq 1$, by Theorem 1.7 we get that $\alpha\left(p_{0}(\cdot)+\alpha_{0}\right) \notin \mathcal{P}\left(\mathbb{R}^{n}\right)$, which gives a counterexample to Question 1.6 .

The paper is organized as follows. Section 2 contains some preliminaries. The proofs of Theorems 1.2, 1.3 and 1.7 are contained in Sections 3, 4 and 5, respectively. 


\section{Preliminaries}

We recall that the Hardy-Littlewood maximal function is defined for $f \in L_{\text {loc }}^{1}\left(\mathbb{R}^{n}\right)$ by

$$
M f(x)=\sup _{Q \ni x} \frac{1}{|Q|} \int_{Q}|f(y)| d y
$$

where the supremum is taken over all cubes $Q$ containing the point $x$. We shall use the classical weak type property of $M$ in the following form (see, e.g., [20, p. 7]):

$$
\left|\left\{x \in \mathbb{R}^{n}: M f(x)>\alpha\right\}\right| \leq \frac{c}{\alpha} \int_{\{x: \mid f(x)>\alpha / 2\}}|f(x)| d x \quad(\alpha>0) .
$$

Recall that the conjugate function $p^{\prime}$ is defined by $\frac{1}{p^{\prime}(x)}+\frac{1}{p(x)}=1$. The following generalized Hölder inequality and a duality relation can be found in [12]:

$$
\begin{aligned}
& \int_{\mathbb{R}^{n}}|f(x) g(x)| d x \leq 2\|f\|_{L^{p(\cdot)}}\|g\|_{L^{p^{\prime}(\cdot)}}, \\
& \|f\|_{L^{p(\cdot)}} \leq \sup _{\|g\|_{L^{p^{\prime}(\cdot)} \leq 1}} \int_{\mathbb{R}^{n}}|f(x) g(x)| d x .
\end{aligned}
$$

By (2.2) and (2.3) it is easy to see that $p \in A$ if and only if

$$
\sup _{Q} \frac{1}{|Q|}\left\|\chi_{Q}\right\|_{L^{p(\cdot)}}\left\|\chi_{Q}\right\|_{L^{p^{\prime}(\cdot)}}<\infty
$$

Definition 2.1. Let $Q_{0}$ be a cube. We say that a weight $w$ (i.e., a nonnegative locally integrable function) satisfies the $A_{\infty}\left(Q_{0}\right)$ condition if there exist constants $\alpha, \beta \in(0,1)$ such that for any cube $Q \subset Q_{0}$ and for any measurable subset $E \subset Q$, $|E|>\alpha|Q|$ implies $w(E)>\beta w(Q)$.

It is well known that the class $A_{\infty}$ can be defined in many equivalent ways. In particular, $w \in A_{\infty}\left(Q_{0}\right)$ if and only if there exist constants $c, \varepsilon>0$ such that for any cube $Q \subset Q_{0}$ and for any measurable subset $E \subset Q$,

$$
\frac{w(E)}{w(Q)} \leq c\left(\frac{|E|}{|Q|}\right)^{\varepsilon}
$$

(see, e.g., [1] where this result is proved in the case $Q_{0}=\mathbb{R}^{n}$; the local case can be treated exactly in the same way).

\section{Proof of Theorem 1.2}

We start with the following lemma due to T. Kopaliani 11. Its proof in 11 is based on some concepts from convex analysis. We give a different and simpler proof here.

Lemma 3.1. Let $p \in A$. Suppose that $|f|_{Q} \geq c_{1}$ and $\|f\|_{L^{p(\cdot)}} \leq c_{2}$, where $c_{1}, c_{2}>0$. Then

$$
\int_{Q}\left(|f|_{Q}\right)^{p(x)} d x \leq c \int_{Q}|f(x)|^{p(x)} d x,
$$

where $c$ depends on $p, c_{1}$ and $c_{2}$. 
Proof. We consider the case $c_{1}=c_{2}=1$; the same proof with trivial modifications works for general $c_{1}$ and $c_{2}$.

Let $\alpha$ be a positive constant satisfying $\int_{Q} \alpha^{p^{\prime}(y)-1} d y=\int_{Q}|f|$. Then

$$
\begin{aligned}
& \int_{Q}\left(|f|_{Q}\right)^{p(x)} d x=\int_{Q}\left(\frac{1}{|Q|} \int_{Q} \alpha^{p^{\prime}(y)-1} d y\right)^{p(x)} d x \\
& =\left(\frac{1}{|Q|} \int_{Q}\left(\frac{1}{|Q|} \int_{Q} \alpha^{p^{\prime}(y)-p^{\prime}(x)} d y\right)^{p(x)-1} d x\right) \int_{Q} \alpha^{p^{\prime}(y)} d y .
\end{aligned}
$$

Since $|f|_{Q} \geq 1$, we have $\alpha \geq 1$. On the other hand, since $\|f\|_{L^{p(\cdot)}} \leq 1$, by (2.2) we get $\int_{Q} \alpha^{p^{\prime}(y)-1} d y \leq 2\left\|\chi_{Q}\right\|_{L^{p^{\prime}(\cdot)}}$. Therefore, $\alpha \leq \frac{c}{\left\|\chi_{Q}\right\|_{L^{p^{\prime}(\cdot)}}}$.

Setting $E_{1}(x)=\left\{y \in Q: p^{\prime}(y)>p^{\prime}(x)\right\}$ and $E_{2}(x)=Q \backslash E_{1}(x)$, and using the above estimates for $\alpha$, we obtain

$$
\begin{aligned}
\int_{Q} \alpha^{p^{\prime}(y)-p^{\prime}(x)} d y & =\int_{E_{1}(x)} \alpha^{p^{\prime}(y)-p^{\prime}(x)} d y+\int_{E_{2}(x)} \alpha^{p^{\prime}(y)-p^{\prime}(x)} d y \\
& \leq c\left\|\chi_{Q}\right\|_{L^{p^{\prime}(\cdot)}}^{p^{\prime}(x)}+|Q| .
\end{aligned}
$$

This, along with $p \in A$, gives

$$
\begin{aligned}
& \left(\frac{1}{|Q|} \int_{Q}\left(\frac{1}{|Q|} \int_{Q} \alpha^{p^{\prime}(y)-p^{\prime}(x)} d y\right)^{p(x)-1} d x\right) \\
& \leq c+c \int_{Q}\left(\frac{\left\|\chi_{Q}\right\|_{L^{p^{\prime}(\cdot)}}}{|Q|}\right)^{p(x)} d x \leq c+c \int_{Q}\left(\frac{1}{\left\|\chi_{Q}\right\|_{L^{p(\cdot)}}}\right)^{p(x)} d x \leq c .
\end{aligned}
$$

Further,

$$
\begin{aligned}
\int_{Q} \alpha^{p^{\prime}(y)} d y & =2 \alpha \int_{Q}|f|-\int_{Q} \alpha^{p^{\prime}(y)} d y \\
& \leq 2 \alpha \int_{\left\{y \in Q: 2 \alpha|f(y)|>\alpha^{p^{\prime}(y)}\right\}}|f(y)| d y \\
& \leq c \int_{Q}|f(y)|^{p(y)} d y .
\end{aligned}
$$

Combining (3.1) with (3.2) and (3.3) completes the proof.

Corollary 3.2. Let $p \in A$. Suppose that $\xi_{1} \leq t \leq \frac{\xi_{2}}{\left\|\chi Q_{0}\right\|_{L^{p(\cdot)}}}$, where $\xi_{1}, \xi_{2}>0$. Then $t^{p(x)} \in A_{\infty}\left(Q_{0}\right)$ with the $A_{\infty}$ constants depending only on $p, \xi_{1}$ and $\xi_{2}$.

Proof. Let $E \subset Q^{\prime} \subset Q_{0}$, where $Q^{\prime}$ is a cube and $|E| \geq\left|Q^{\prime}\right| / 2$. Then $f=t \chi_{E}$ satisfies the conditions of Lemma 3.1 with $Q=Q^{\prime}, c_{1}=\xi_{1} / 2$ and $c_{2}=\xi_{2}$. Hence,

$$
\frac{1}{2^{p_{+}}} \int_{Q^{\prime}} t^{p(x)} d x \leq c \int_{E} t^{p(x)} d x
$$

which proves the $A_{\infty}\left(Q_{0}\right)$ condition.

Proof of Theorem 1.2. For each integer $k$ set

$$
\Omega_{k}=\left\{x \in \mathbb{R}^{n}: M f(x)>3^{n k}\right\}
$$

and $D_{k}=\Omega_{k} \backslash \Omega_{k+1}$. Let $F_{k}$ be an arbitrary compact subset of $D_{k}$. 
Fix a function $\varphi \geq 0$ supported in $E$ and such that $\|\varphi\|_{L^{p^{\prime}(\cdot)}} \leq 1$. We are going to show that

$$
\int_{\bigcup_{k=-\infty}^{\infty} F_{k}}(M f) \varphi d x \leq c\|f\|_{L^{p(\cdot)}},
$$

where $c=c(p, n, E)$. By (2.3) and by the standard limiting argument, this inequality readily gives the desired result.

By the Vitali covering lemma, there exists a finite collection of pairwise disjoint cubes $\left\{Q_{j}^{k}\right\}_{j \geq 1}$ such that $F_{k} \subset \bigcup_{j} 3 Q_{j}^{k}$ and $|f|_{Q_{j}^{k}}>3^{n k}$. Let $E_{1}^{k}=3 Q_{1}^{k} \cap F_{k}$ and $E_{j}^{k}=\left(3 Q_{j}^{k} \backslash \bigcup_{s<j} 3 Q_{s}^{k}\right) \cap F_{k}, j>1$. Note that the sets $E_{j}^{k}$ are pairwise disjoint and $\bigcup_{j} E_{j}^{k}=F_{k}$.

Using the above definitions and (2.2), we get

$$
\begin{aligned}
\int_{\bigcup_{k=-\infty}^{\infty} F_{k}}(M f) \varphi d x & \leq 3^{n} \sum_{k=-\infty}^{\infty} \sum_{j}|f|_{Q_{j}^{k}} \int_{E_{j}^{k}} \varphi \\
& =3^{n} \int_{\mathbb{R}^{n}}|f| T \varphi d x \leq 2 \cdot 3^{n}\|f\|_{L^{p(\cdot)}}\|T \varphi\|_{L^{p^{\prime}(\cdot)}},
\end{aligned}
$$

where

$$
T \varphi(x)=\sum_{k=-\infty}^{\infty} \sum_{j}\left(\frac{1}{\left|Q_{j}^{k}\right|} \int_{E_{j}^{k}} \varphi\right) \chi_{Q_{j}^{k}}(x) .
$$

Hence, in order to prove (3.4), it suffices to show that

$$
\|T \varphi\|_{L^{p^{\prime}(\cdot)}} \leq c(p, n, E) .
$$

Let $\alpha_{j, k}(\varphi)=\frac{1}{\left|Q_{j}^{k}\right|} \int_{E_{j}^{k}} \varphi$ and

$$
T_{l} \varphi(x)=\sum_{k=-\infty}^{\infty} \sum_{j} \alpha_{j, k}(\varphi) \chi_{Q_{j}^{k} \cap D_{k+l}}(x) \quad(l=0,1, \ldots) .
$$

Note that $Q_{j}^{k} \subset \Omega_{k}=\bigcup_{l=0}^{\infty} D_{k+l}$, and hence $T \varphi(x)=\sum_{l=0}^{\infty} T_{l} \varphi(x)$. Also, since the sets $Q_{j}^{k} \cap D_{k+l}$ are pairwise disjoint, we have

$$
\int_{\mathbb{R}^{n}}\left(T_{l} \varphi\right)^{p^{\prime}(x)} d x=\sum_{k=-\infty}^{\infty} \sum_{j} \int_{Q_{j}^{k} \cap D_{k+l}} \alpha_{j, k}(\varphi)^{p^{\prime}(x)} d x .
$$

We divide the last sum into two sums corresponding the indices $\mathcal{I}_{1}=\{(j, k)$ : $\left.\alpha_{j, k}(\varphi)>1\right\}$ and $\mathcal{I}_{2}=\left\{(j, k): \alpha_{j, k}(\varphi) \leq 1\right\}$.

Suppose first that $(j, k) \in \mathcal{I}_{1}$. By (2.2) and by condition $A$,

$$
\begin{aligned}
\alpha_{j, k}(\varphi) & \leq \frac{2}{\left|Q_{j}^{k}\right|}\left\|\chi_{E_{j}^{k}}\right\|_{L^{p(\cdot)}} \leq \frac{2}{\left|Q_{j}^{k}\right|}\left\|\chi_{3 Q_{j}^{k}}\right\|_{L^{p(\cdot)}} \\
& \leq \frac{c}{\left|Q_{j}^{k}\right|}\left\|\chi_{Q_{j}^{k}}\right\|_{L^{p(\cdot)}} \leq \frac{c}{\left\|\chi_{Q_{j}^{k}}\right\|_{L^{p^{\prime}(\cdot)}}}
\end{aligned}
$$

(we have used the fact that condition $A$ implies the following "doubling" property: there exists $c>0$ such that $\left\|\chi_{2 Q}\right\|_{L^{p(\cdot)}} \leq c\left\|\chi_{Q}\right\|_{L^{p(\cdot)}}$ for any cube $Q$ ). Hence, by 
Corollary $3.2 \alpha_{j, k}(\varphi)^{p^{\prime}(x)} \in A_{\infty}\left(Q_{j}^{k}\right)$. From this and from Lemma 3.1

$$
\begin{aligned}
\int_{Q_{j}^{k} \cap D_{k+l}} \alpha_{j, k}(\varphi)^{p^{\prime}(x)} d x & \leq c\left(\frac{\left|Q_{j}^{k} \cap D_{k+l}\right|}{\left|Q_{j}^{k}\right|}\right)^{\varepsilon} \int_{Q_{j}^{k}} \alpha_{j, k}(\varphi)^{p^{\prime}(x)} d x \\
& \leq c\left(\frac{\left|Q_{j}^{k} \cap D_{k+l}\right|}{\left|Q_{j}^{k}\right|}\right)^{\varepsilon} \int_{E_{j}^{k}} \varphi(x)^{p^{\prime}(x)} d x .
\end{aligned}
$$

Assume now that $(j, k) \in \mathcal{I}_{2}$. Then

$$
\begin{aligned}
\int_{Q_{j}^{k} \cap D_{k+l}} \alpha_{j, k}(\varphi)^{p^{\prime}(x)} d x & \leq \int_{Q_{j}^{k} \cap D_{k+l}} \alpha_{j, k}(\varphi) d x \\
& =\frac{\left|Q_{j}^{k} \cap D_{k+l}\right|}{\left|Q_{j}^{k}\right|} \int_{E_{j}^{k}} \varphi .
\end{aligned}
$$

Let us show now that for each $Q_{j}^{k}$,

$$
\left|Q_{j}^{k} \cap D_{k+l}\right| \leq 3^{n(3-l)}\left|Q_{j}^{k}\right| \quad(l \geq 4) .
$$

Indeed, let $x \in Q_{j}^{k}$ and let $Q^{\prime}$ be an arbitrary cube such that $x \in Q^{\prime}$. Observe that either $Q^{\prime} \subset 3 Q_{j}^{k}$ or $Q_{j}^{k} \subset 3 Q^{\prime}$. If the second inclusion holds, then $3 Q^{\prime} \cap D_{k} \neq \emptyset$, and hence

$$
|f|_{Q^{\prime}} \leq 3^{n}|f|_{3 Q^{\prime}} \leq 3^{n} 3^{n(k+1)} \leq 3^{n(k+l)} \quad(l \geq 2) .
$$

Therefore, if $|f|_{Q^{\prime}}>3^{n(k+l)}$, then $Q^{\prime} \subset 3 Q_{j}^{k}$. From this and from the weak type $(1,1)$ property of $M$, we get

$$
\begin{aligned}
\left|Q_{j}^{k} \cap D_{k+l}\right| & \leq\left|\left\{x \in Q_{j}^{k}: M\left(f \chi_{3 Q_{j}^{k}}\right)(x)>3^{n(k+l)}\right\}\right| \\
& \leq \frac{3^{n}}{3^{n(k+l)}} \int_{3 Q_{j}^{k}}|f| \leq \frac{9^{n}\left|Q_{j}^{k}\right|}{3^{n(k+l)}}|f|_{3 Q_{j}^{k}} \leq \frac{9^{n}}{3^{n(l-1)}}\left|Q_{j}^{k}\right|,
\end{aligned}
$$

proving (3.8).

Combining (3.6), (3.7) and (3.8), we get (for $0 \leq l \leq 3$ we use a trivial estimate $\left.\left|Q_{j}^{k} \cap D_{k+l}\right| \leq\left|Q_{j}^{k}\right|\right)$

$$
\begin{aligned}
& \sum_{k=-\infty}^{\infty} \sum_{j} \int_{Q_{j}^{k} \cap D_{k+l}} \alpha_{j, k}(\varphi)^{p^{\prime}(x)} d x \\
& \leq \sum_{(j, k) \in \mathcal{I}_{1}} c 3^{n \varepsilon(3-l)} \int_{E_{j}^{k}} \varphi(x)^{p^{\prime}(x)} d x+\sum_{(j, k) \in \mathcal{I}_{2}} 3^{n(3-l)} \int_{E_{j}^{k}} \varphi \\
& \leq c 3^{n \varepsilon(3-l)}\left(\int_{\mathbb{R}^{n}} \varphi(x)^{p^{\prime}(x)} d x+\|\varphi\|_{L^{1}}\right) .
\end{aligned}
$$

However, $\int_{\mathbb{R}^{n}} \varphi(x)^{p^{\prime}(x)} d x \leq 1$, and by (2.2),

$$
\|\varphi\|_{L^{1}}=\int_{E} \varphi \leq 2\left\|\chi_{E}\right\|_{L^{p(\cdot)}} .
$$

Therefore,

which easily implies

$$
\int_{\mathbb{R}^{n}}\left(T_{l} \varphi\right)^{p^{\prime}(x)} d x \leq c\left\|\chi_{E}\right\|_{L^{p(\cdot)}} 3^{n \varepsilon(3-l)},
$$

$$
\left\|T_{l} f\right\|_{L^{p^{\prime}(\cdot)}} \leq c(p, n, E)\left(3^{n \varepsilon /\left(p^{\prime}\right)_{+}}\right)^{-l} \quad(l=0,1, \ldots) .
$$


This estimate, along with

$$
\|T f\|_{L^{p^{\prime}(\cdot)}} \leq \sum_{l=0}^{\infty}\left\|T_{l} f\right\|_{L^{p^{\prime}(\cdot)}}
$$

proves (3.5), and therefore the proof is complete.

Remark 3.3. The proof of Theorem 1.2 shows that

$$
\left\|(M f) \chi_{E}\right\|_{L^{p(\cdot)}} \leq c(p, n) c(E)\|f\|_{L^{p(\cdot)}},
$$

where $c(E)=\max \left(1,\left\|\chi_{E}\right\|_{L^{p(\cdot)}}^{1 /\left(p^{\prime}\right)_{-}}\right)$.

Remark 3.4. Theorem 1.2 easily implies the following result due to T. Kopaliani [11 mentioned in the Introduction: if $p$ is a constant outside some ball and $p \in A$, then $M$ is bounded on $L^{p(\cdot)}$. Indeed, let $p(x)=p_{0}$ on $B^{c}$, and let $\|f\|_{L^{p(\cdot)}}=1$. Then Theorem 1.2 with $E=2 B$ gives

$$
\int_{\mathbb{R}^{n}}(M f)^{p(x)} d x \leq c+\int_{(2 B)^{c}}(M f)^{p_{0}} d x .
$$

Next, setting $f_{1}=f \chi_{B}$ and $f_{2}=f-f_{1}$, we get

$$
\int_{(2 B)^{c}}(M f)^{p_{0}} d x \leq 2^{p_{0}-1}\left(\int_{(2 B)^{c}}\left(M f_{1}\right)^{p_{0}} d x+\int_{(2 B)^{c}}\left(M f_{2}\right)^{p_{0}} d x\right) .
$$

By the Hardy-Littlewood maximal theorem,

$$
\int_{(2 B)^{c}}\left(M f_{2}\right)^{p_{0}} d x \leq c \int_{B^{c}}|f|^{p_{0}} d x \leq c .
$$

Finally, by (2.2), $\int_{B}|f| \leq c_{B}$, and hence

$$
\int_{(2 B)^{c}}\left(M f_{1}\right)^{p_{0}} d x \leq c\left(\int_{(2 B)^{c}} \frac{1}{\left|x-x_{0}\right|^{p_{0}}} d x\right)\left(\int_{B}|f|\right)^{p_{0}} \leq c,
$$

where $x_{0}$ is the center of $B$. We have proved that $\int(M f)^{p(x)} d x \leq c$ whenever $\|f\|_{L^{p(\cdot)}}=1$, which means the boundedness of $M$ on $L^{p(\cdot)}$.

\section{Proof of Theorem 1.3}

The necessity part of Theorem 1.3 follows immediately from the fact that

$$
|f|_{Q} \chi_{Q}(x) \leq M f(x)
$$

for any cube $Q$. In proving the sufficiency part it will be more convenient to work with the maximal function defined with respect to balls, so we shall assume in this section that

$$
M f(x)=\sup _{B \ni x} \frac{1}{|B|} \int_{B}|f(y)| d y,
$$

where the supremum is taken over all balls $B$ containing the point $x$.

Proof of the sufficiency part of Theorem 1.3. Let $p$ be radially decreasing and $p \in$ $A$. The weak type $(p(\cdot), p(\cdot))$ of $M$ means that there exists a constant $c>0$ such that for any $f \in L^{p(\cdot)}$ with $\|f\|_{L^{p(\cdot)}}=1$ one has

$$
\sup _{\alpha>0} \int_{\{x: M f(x)>\alpha\}} \alpha^{p(x)} d x \leq c .
$$


Fix an $f \in L^{p(\cdot)}$ with $\|f\|_{L^{p(\cdot)}}=1$. Observe that the case corresponding to $\alpha \geq 1$ follows easily from Theorem 1.2. Indeed, let $E=\{x: M f(x)>1\}$. By (2.1),

$$
|E| \leq c \int_{\{x:|f(x)|>1 / 2\}}|f(x)| d x \leq c \int_{\mathbb{R}^{n}}|f(x)|^{p(x)} d x \leq c .
$$

Therefore, $\left\|\chi_{E}\right\|_{L^{p(\cdot)}} \leq c$, and by (3.9) we get

$$
\int_{\{x: M f(x)>\alpha\}} \alpha^{p(x)} d x \leq c \int_{E}(M f)^{p(x)} d x \leq c \quad(\alpha \geq 1) .
$$

The case when $\alpha<1$ is more complicated. We are going to show that

$$
\sup _{0<\alpha<1} \int_{\left\{x: M f(x)>2^{n+1} \alpha\right\}} \alpha^{p(x)} d x \leq c .
$$

Clearly, this estimate, along with (4.2), would imply (4.1). We start by defining several auxiliary functions.

Given a ball $B$, denote by $\widetilde{B}$ the ball of the same radius centered at the origin. Suppose that $|B| \geq 1$. Then $\left\|\chi_{\tilde{B}}\right\|_{L^{p^{\prime}(\cdot)}} \geq 1$. Since $p(x)$ is radially decreasing, $p^{\prime}(x)$ is radially increasing, and thus

$$
\int_{B}\left(\frac{1}{\left\|\chi_{\widetilde{B}}\right\|_{L^{p^{\prime}(\cdot)}}}\right)^{p^{\prime}(x)} d x \leq \int_{\widetilde{B}}\left(\frac{1}{\left\|\chi_{\widetilde{B}}\right\|_{L^{p^{\prime}(\cdot)}}}\right)^{p^{\prime}(x)} d x=1 .
$$

Hence,

$$
\left\|\chi_{B}\right\|_{L^{p^{\prime}(\cdot)}} \leq\left\|\chi_{\widetilde{B}}\right\|_{L^{p^{(\cdot)}}}
$$

and therefore for $h>1$ we have

$$
\psi(h) \equiv \frac{1}{h} \sup _{|B|=h}\left\|\chi_{B}\right\|_{L^{\left.p^{(} \cdot\right)}}=\frac{1}{h}\left\|\chi_{\left\{|x| \leq\left(h / v_{n}\right)^{1 / n}\right\}}\right\|_{L^{p^{\prime}(\cdot)}},
$$

where $v_{n}$ is the volume of the unit ball. Observe that if $|B| \geq 1$, then

$$
|B|^{1 /\left(p^{\prime}\right)_{+}} \leq\left\|\chi_{B}\right\|_{L^{p^{\prime}(\cdot)}} \leq|B|^{1 /\left(p^{\prime}\right)_{-}} .
$$

From this and since $\left(p^{\prime}\right)_{+}=\left(p_{-}\right)^{\prime}$ and $\left(p^{\prime}\right)_{-}=\left(p_{+}\right)^{\prime}$, we get

$$
(1 / h)^{1 / p_{-}} \leq \psi(h) \leq(1 / h)^{1 / p_{+}} \quad(h>1) .
$$

Now, for $0<\alpha<1$ define

$$
\varphi(\alpha)=\sup \{h>1: \psi(h)>\alpha\} .
$$

By (4.4),

$$
(1 / \alpha)^{p_{-}} \leq \varphi(\alpha) \leq(1 / \alpha)^{p_{+}} \quad(0<\alpha<1)
$$

Further, setting $B_{\alpha}=\left\{x:|x| \leq\left(\varphi(\alpha) / v_{n}\right)^{1 / n}\right\}$, we have

$$
\frac{\left\|\chi_{B_{\alpha}}\right\|_{L^{p^{\prime}(\cdot)}}}{\left|B_{\alpha}\right|}=\psi(\varphi(\alpha))=\alpha .
$$

Hence, since $p \in A$, we obtain $\alpha\left\|\chi_{B_{\alpha}}\right\|_{L^{p(\cdot)}} \leq c$, or equivalently,

$$
\int_{B_{\alpha}} \alpha^{p(x)} d x \leq c
$$

(we have used an obvious fact that the definitions of class $A$ in terms of cubes and balls are equivalent). 
Setting $S_{k}(\alpha)=(k+1) B_{\alpha} \backslash k B_{\alpha}$ and using (4.6), we get

$$
\int_{\left\{x: M f(x)>2^{n+1} \alpha\right\}} \alpha^{p(x)} d x \leq c+\sum_{k=1}^{\infty} \int_{S_{k}(\alpha) \cap\left\{M f>2^{n+1} \alpha\right\}} \alpha^{p(x)} d x .
$$

Now set $\widetilde{S}_{k}(\alpha)=(k+3 / 2) B_{\alpha} \backslash(k-1 / 2) B_{\alpha}$. Note that

$$
\bigcup_{x \in S_{k}(\alpha)}\left\{B: x \in B,|B| \leq\left|B_{\alpha} / 2\right|\right\} \subset \widetilde{S}_{k}(\alpha) .
$$

Further, if $|B|>\left|B_{\alpha} / 2\right|$, then $|2 B|>\varphi(\alpha)$, and hence, by the definition of $\varphi$ and by (2.2) we get

$$
\frac{1}{|B|} \int_{B}|f| \leq \frac{2^{n}}{|2 B|} \int_{2 B}|f| \leq 2^{n+1} \frac{\left\|\chi_{2 B}\right\|_{L^{p^{\prime}(\cdot)}}}{|2 B|} \leq 2^{n+1} \alpha .
$$

Therefore,

$$
S_{k}(\alpha) \cap\left\{M f>2^{n+1} \alpha\right\} \subset\left\{M\left(f \chi_{\widetilde{S}_{k}(\alpha)}\right)>2^{n+1} \alpha\right\} .
$$

Hence, setting $\gamma_{\alpha}=\left(\varphi(\alpha) / v_{n}\right)^{1 / n}$ and using the fact that $p$ is radially decreasing along with (2.1), we get (recall that $p(x)=\rho(|x|)$ )

$$
\begin{aligned}
& \int_{S_{k}(\alpha) \cap\left\{M f>2^{n+1} \alpha\right\}} \alpha^{p(x)} d x \leq \alpha^{\rho\left((k+1) \gamma_{\alpha}\right)}\left|\left\{M\left(f \chi_{\widetilde{S}_{k}(\alpha)}\right)>2^{n+1} \alpha\right\}\right| \\
& \leq c \alpha^{\rho\left((k+1) \gamma_{\alpha}\right)-1} \int_{\widetilde{S}_{k}(\alpha) \cap\left\{|f|>2^{n} \alpha\right\}}|f| \\
& \leq c \alpha^{\rho\left((k+1) \gamma_{\alpha}\right)-\rho\left((k-1 / 2) \gamma_{\alpha}\right)} \int_{\widetilde{S}_{k}(\alpha)}|f(x)|^{p(x)} d x .
\end{aligned}
$$

It is easy to see that $\sum_{k=1}^{\infty} \chi_{\widetilde{S}_{k}(\alpha)}(x) \leq 2$, and hence

$$
\sum_{k=1}^{\infty} \int_{\widetilde{S}_{k}(\alpha)}|f(x)|^{p(x)} d x \leq 2 .
$$

Combining this with (4.7) and (4.8), we have that in order to prove (4.3), it suffices to show that

$$
\sup _{0<\alpha<1} \alpha^{\rho\left((k+1) \gamma_{\alpha}\right)-\rho\left((k-1 / 2) \gamma_{\alpha}\right)} \leq c,
$$

where $c$ does not depend on $k$.

Let $\xi_{1}=\left((k+3 / 2) \gamma_{\alpha}, 0, \ldots, 0\right), \xi_{2}=\left((k-1) \gamma_{\alpha}, 0, \ldots, 0\right)$, and let $B_{1}$ and $B_{2}$ be the balls of radius $\gamma_{\alpha} / 2$ centered at $\xi_{1}$ and $\xi_{2}$, respectively. Next, let $\xi_{3}=\left(\xi_{1}+\xi_{2}\right) / 2$ and let $B_{3}$ be the ball centered at $\xi_{3}$ of radius $2 \gamma_{\alpha}$. Then the balls $B_{1}, B_{2}$ and $B_{3}$ satisfy the following properties:

(i) $\inf _{x \in B_{1}}|x|=(k+1) \gamma_{\alpha}$ and $\sup _{x \in B_{2}}|x|=(k-1 / 2) \gamma_{\alpha}$;

(ii) $\left|B_{1}\right|=\left|B_{2}\right|=\varphi(\alpha) / 2^{n}$;

(iii) $B_{1}, B_{2} \subset B_{3}$ and $\left|B_{3}\right|=2^{n} \varphi(\alpha)$.

If the supremum in (4.9) is taken over $2^{-n / p_{-}}<\alpha<1$, then the bound is trivial. Hence, one can assume that $\alpha \leq 2^{-n / p_{-}}$. Then, by (4.5), $\left|B_{1}\right|=\left|B_{2}\right| \geq 1$, and therefore,

$$
\varphi(\alpha) 2^{-n}\left(\frac{1}{\left\|\chi_{B_{1}}\right\|_{L^{p(\cdot)}}}\right)^{\rho\left((k+1) \gamma_{\alpha}\right)} \leq \int_{B_{1}}\left(\frac{1}{\left\|\chi_{B_{1}}\right\|_{L^{p(\cdot)}}}\right)^{p(x)} d x=1
$$


and

$$
\varphi(\alpha) 2^{-n}\left(\frac{1}{\left\|\chi_{B_{2}}\right\|_{L^{p^{\prime}(\cdot)}}}\right)^{\rho^{\prime}\left((k-1 / 2) \gamma_{\alpha}\right)} \leq \int_{B_{2}}\left(\frac{1}{\left\|\chi_{B_{2}}\right\|_{L^{p^{\prime}(\cdot)}}}\right)^{p^{\prime}(x)} d x=1 .
$$

Using these estimates and condition $A$, we get

$$
\begin{aligned}
\varphi(\alpha)^{1 / \rho\left((k+1) \gamma_{\alpha}\right)+1 / \rho^{\prime}\left((k-1 / 2) \gamma_{\alpha}\right)} & \leq c\left\|\chi_{B_{1}}\right\|_{L^{p(\cdot)}}\left\|\chi_{B_{2}}\right\|_{L^{p^{\prime}(\cdot)}} \\
& \leq c\left\|\chi_{B_{3}}\right\|_{L^{p(\cdot)}}\left\|\chi_{B_{3}}\right\|_{L^{p^{\prime}(\cdot)}} \leq c \varphi(\alpha) .
\end{aligned}
$$

Hence

and thus

$$
\varphi(\alpha)^{1 / \rho\left((k+1) \gamma_{\alpha}\right)-1 / \rho\left((k-1 / 2) \gamma_{\alpha}\right)} \leq c,
$$

$$
\varphi(\alpha)^{\frac{1}{\left(\rho_{+}\right)^{2}} \rho\left((k-1 / 2) \gamma_{\alpha}\right)-\rho\left((k+1) \gamma_{\alpha}\right)} \leq c .
$$

Combining this with the left-hand side of (4.5) proves (4.9), and therefore the theorem is proved.

\section{Proof of Theorem 1.7}

We start with the following characterization of condition $A$ for big cubes for radially decreasing $p$.

Lemma 5.1. Let $p$ be a radially decreasing function $(p(x)=\rho(|x|))$ with $p_{-}>1$ and $p_{+}<\infty$. Then

$$
\sup _{|Q| \geq 1} \frac{\left\|\chi_{Q}\right\|_{L^{p(\cdot)}}\left\|\chi_{Q}\right\|_{L^{p^{\prime}(\cdot)}}}{|Q|}<\infty
$$

if and only if

$$
\sup _{t \geq 1} \int_{0}^{1} t^{n\left(\frac{\rho(\xi t)-\rho(t)}{\rho(t)(\rho(\xi t)-1)}\right)} \xi^{n-1} d \xi<\infty .
$$

Proof. Given $t>0$, let $B_{t}$ be the ball centered at the origin of radius $t$. Observe that (5.2) is equivalent to

$$
\sup _{t \geq 1}\left\|\chi_{B_{t}}\right\|_{L^{p^{\prime}(\cdot)}} t^{-n / \rho^{\prime}(t)}<\infty .
$$

Indeed, (5.3) holds if and only if

$$
\sup _{t \geq 1} \int_{B_{t}}\left(\frac{1}{t^{n}}\right)^{\frac{p^{\prime}(x)}{\rho^{\prime}(t)}} d x<\infty .
$$

However,

$$
\begin{aligned}
& \int_{B_{t}}\left(\frac{1}{t^{n}}\right)^{\frac{p^{\prime}(x)}{\rho^{\prime}(t)}} d x=\omega_{n-1} \int_{0}^{t}\left(\frac{1}{t^{n}}\right)^{\frac{\rho^{\prime}(\xi)}{\rho^{\prime}(t)}} \xi^{n-1} d \xi \\
& =\omega_{n-1} \int_{0}^{1}\left(\frac{1}{t^{n}}\right)^{\frac{\rho^{\prime}(\xi t)}{\rho^{\prime}(t)}-1} \xi^{n-1} d \xi=\omega_{n-1} \int_{0}^{1} t^{n\left(\frac{\rho(\xi t)-\rho(t)}{\rho(t)(\rho(\xi t)-1)}\right)} \xi^{n-1} d \xi,
\end{aligned}
$$

where $\omega_{n-1}$ is the surface area of the unit sphere in $\mathbb{R}^{n}$.

Assume now that (5.1) holds. By (2.2) and (2.3), this is equivalent to

$$
|f|_{Q}\left\|\chi_{Q}\right\|_{L^{p(\cdot)}} \leq c\left\|f \chi_{Q}\right\|_{L^{p(\cdot)}}
$$


for any locally integrable $f$ and any cube with $|Q| \geq 1$. In particular, setting $f=\chi_{Q / 2}$, we get

$$
\left\|\chi_{Q}\right\|_{L^{p(\cdot)}} \leq c\left\|\chi_{Q / 2}\right\|_{L^{p(\cdot)}} \quad(|Q| \geq 1) .
$$

Let $Q_{t}$ be the smallest cube containing $B_{t}$. From (5.4), for $t \geq 1$ we get

$$
\begin{aligned}
\left(2^{n}\left(2^{n}-1\right) t^{n}\right)^{1 / \rho(t)} & =\left|Q_{2 t} \backslash Q_{t}\right|^{\frac{1}{p_{+}\left(Q_{2 t} \backslash Q_{t}\right)}} \\
& \leq\left\|\chi_{Q_{2 t} \backslash Q_{t}}\right\|_{L^{p(\cdot)}} \leq\left\|\chi_{Q_{2 t}}\right\|_{L^{p(\cdot)}} \leq c\left\|\chi_{Q_{t}}\right\|_{L^{p(\cdot)}}
\end{aligned}
$$

(we use the notion $p_{-}(E)=\underset{x \in E}{\operatorname{essinf}} p(x)$ and $p_{+}(E)=\underset{x \in E}{\operatorname{ess} \sup } p(x)$ ). Therefore, by (5.1),

$$
\left\|\chi_{B_{t}}\right\|_{L^{p^{\prime}(\cdot)}} \leq\left\|\chi_{Q_{t}}\right\|_{L^{p^{\prime}(\cdot)}} \leq c \frac{\left|Q_{t}\right|}{\left\|\chi_{Q_{t}}\right\|_{L^{p(\cdot)}}} \leq c t^{n / \rho^{\prime}(t)}
$$

which proves (5.3) and so (5.2).

Suppose now that (5.2) holds. Let $Q=\prod_{i=1}^{n}\left(a_{i}, a_{i}+h\right)$, where $h \geq 1$. Denote $\alpha=\max _{1 \leq i \leq n}\left|a_{i}\right|$, and assume that $\alpha \leq 2 h$. Then it is easy to see that $Q \subset B_{3 \sqrt{n} h}$. Next, since $p$ is radially decreasing,

$$
\left\|\chi_{B_{3 \sqrt{n} h}}\right\|_{L^{p(\cdot)}} \leq\left|B_{3 \sqrt{n} h}\right|^{1 / \rho(3 \sqrt{n} h)} .
$$

From this and from (5.3),

$$
\begin{aligned}
\frac{\left\|\chi_{Q}\right\|_{L^{p(\cdot)}}\left\|\chi_{Q}\right\|_{L^{p^{\prime}(\cdot)}}}{|Q|} \leq \frac{\left\|\chi_{B_{3 \sqrt{n} h}}\right\|_{L^{p(\cdot)}}\left\|\chi_{B_{3 \sqrt{n} h}}\right\|_{L^{p^{\prime}(\cdot)}}}{h^{n}} \\
\leq c\left\|\chi_{B_{3 \sqrt{n} h}}\right\|_{L^{p^{\prime}(\cdot)}} h^{-n / \rho^{\prime}(3 \sqrt{n} h)} \leq c .
\end{aligned}
$$

It remains to consider the case when $\alpha>2 h$. In this case,

$$
\sup _{x \in Q}|x| \leq \frac{3 \sqrt{n}}{2} \alpha \quad \text { and } \quad \inf _{x \in Q}|x| \geq \frac{1}{2} \alpha
$$

and therefore,

$$
p_{+}(Q) \leq \rho(\alpha / 2) \quad \text { and } \quad p_{-}(Q) \geq \rho(3 \sqrt{n} \alpha / 2) .
$$

Next, since $|Q| \geq 1$,

$$
\left\|\chi_{Q}\right\|_{L^{p(\cdot)}} \leq|Q|^{1 / p_{-}(Q)} \quad \text { and } \quad\left\|\chi_{Q}\right\|_{L^{p^{(\cdot)}}} \leq|Q|^{1-1 / p_{+}(Q)} .
$$

Combining these estimates yields

$$
\begin{aligned}
\frac{\left\|\chi_{Q}\right\|_{L^{p(\cdot)}}\left\|\chi_{Q}\right\|_{L^{p^{(\cdot)}}}}{|Q|} & \leq|Q|^{\frac{p_{+}(Q)-p_{-}(Q)}{p_{+}(Q) p_{-}(Q)}} \\
& \leq c \alpha^{n(\rho(\alpha / 2)-\rho(3 \sqrt{n} \alpha / 2)) / p_{-}^{2}} .
\end{aligned}
$$

But it follows from (5.2) that for $t \geq 1$,

$$
t^{\rho(\xi t)-\rho(t)} \leq c \quad(0<\xi<1),
$$

where $c$ depends only on $\xi$ and $p$. Indeed, since $\rho$ is nonincreasing, by (5.2) we get

$$
t^{\frac{n}{\rho+\left(\rho_{+}-1\right)}}(\rho(\xi t)-\rho(t)) \frac{\xi^{n}}{n} \leq \int_{0}^{\xi} t^{n\left(\frac{\rho(\tau t)-\rho(t)}{\rho(t)(\rho(\tau t)-1)}\right)} \tau^{n-1} d \tau \leq c .
$$

Since $\alpha>2 h \geq 2$, we obtain that the right-hand side of (5.5) is bounded, which completes the proof. 
Proof of Theorem 1.7. Let $E=\bigcup_{k=1}^{\infty}\left(e^{k^{3}}, e^{k^{3} e^{1 / k^{2}}}\right)$ and

$$
p_{0}(x)=\int_{|x|}^{\infty} \frac{1}{\tau \log \tau} \chi_{E}(\tau) d \tau .
$$

Let us show that $p_{0}$ is a pointwise multiplier for $B M O(\mathbb{R})$. This is just a combination of several known facts. First, it was proved in [15] that $p$ is a pointwise multiplier for $B M O(\mathbb{R})$ if and only if $p \in L^{\infty}(\mathbb{R})$ and

$$
\sup _{I} \frac{\ell(I)}{|I|^{2}} \int_{I} \int_{I}|p(x)-p(y)| d x d y<\infty
$$

where the supremum is taken over all intervals $I \subset \mathbb{R}$,

$$
\ell(I)=\log \left(e+\max \left(|I|,|I|^{-1},\left|\operatorname{cen}_{I}\right|\right)\right)
$$

and $\operatorname{cen}_{I}$ denotes the center of $I$. Next, in proving [13, Proposition 4.2] it contains the proof of the fact that $g(x)=(\log \log |x|) \chi_{\{|x| \geq e\}}(x)$ satisfies (5.6). But $p_{0} \in L^{\infty}$, and it is easy to see that for all $x, y \in \mathbb{R}$,

$$
\left|p_{0}(x)-p_{0}(y)\right| \leq|g(x)-g(y)| \text {. }
$$

Therefore, $p_{0}$ satisfies (5.6), which proves that $p_{0}$ is a pointwise multiplier for $B M O(\mathbb{R})$.

Let $p_{q, \delta}(x)=q+\delta p_{0}(x)$, and assume that $q(q-1) \leq \delta$. Let us show that $p_{q, \delta} \notin A$. By Lemma 5.1, it suffices to prove that

$$
\sup _{t \geq 1} \int_{0}^{1} t^{\frac{\rho(\xi t)-\rho(t)}{\rho(t)(\rho(\xi t)-1)}} d \xi=\infty .
$$

Denote $\alpha_{k}=e^{k^{3}}$ and $\beta_{k}=e^{k^{3} e^{1 / k^{2}}}$. We have

$$
\int_{0}^{1} \beta_{k}^{\frac{\rho\left(\xi \beta_{k}\right)-p\left(\beta_{k}\right)}{\rho\left(\beta_{k}\right)\left(\rho\left(\xi \beta_{k}\right)-1\right)}} d \xi \geq \int_{\alpha_{k} / \beta_{k}}^{1} \beta_{k}^{\frac{\delta\left(\log \log \beta_{k}-\log \log \xi \beta_{k}\right)}{\rho\left(\beta_{k}\right)\left(\rho\left(\alpha_{k}\right)-1\right)}} d \xi .
$$

There exists a sequence $\left\{\varepsilon_{k}\right\}$ such that $\varepsilon_{k} \rightarrow 0$ as $k \rightarrow \infty$ and

$$
\log \log \beta_{k}-\log \log \xi \beta_{k}=\log \left(\frac{\log (1 / \xi)}{\log \left(\xi \beta_{k}\right)}+1\right) \geq\left(1-\varepsilon_{k}\right) \frac{\log (1 / \xi)}{\log \left(\xi \beta_{k}\right)}
$$

for any $\xi \in\left(\alpha_{k} / \beta_{k}, 1\right)$. Hence,

$$
\beta_{k}^{\log \log \beta_{k}-\log \log \xi \beta_{k}} \geq \beta_{k}^{\left(1-\varepsilon_{k}\right) \frac{\log (1 / \xi)}{\log \left(\xi \beta_{k}\right)}}=(1 / \xi)^{\left(1-\varepsilon_{k}\right) \frac{\log \beta_{k}}{\log \xi \beta_{k}}} \geq(1 / \xi)^{\left(1-\varepsilon_{k}\right)} .
$$

From this,

$$
\int_{\alpha_{k} / \beta_{k}}^{1} \beta_{k}^{\frac{\delta\left(\log \log \beta_{k}-\log \log \xi \beta_{k}\right)}{\rho\left(\beta_{k}\right)\left(\rho\left(\alpha_{k}\right)-1\right)}} d \xi \geq \int_{\alpha_{k} / \beta_{k}}^{1} \xi^{-\frac{\delta\left(1-\varepsilon_{k}\right)}{\rho\left(\beta_{k}\right)\left(\rho\left(\alpha_{k}\right)-1\right)}} d \xi .
$$

Since $\alpha_{k} / \beta_{k} \rightarrow 0$ and $\frac{\delta\left(1-\varepsilon_{k}\right)}{\rho\left(\beta_{k}\right)\left(\rho\left(\alpha_{k}\right)-1\right)} \rightarrow \frac{\delta}{q(q-1)} \geq 1$, we have

$$
\int_{\alpha_{k} / \beta_{k}}^{1} \xi^{-\frac{\delta\left(1-\varepsilon_{k}\right)}{\rho\left(\beta_{k}\right)\left(\rho\left(\alpha_{k}\right)-1\right)}} d \xi \rightarrow \infty \quad \text { as } \quad k \rightarrow \infty .
$$

Indeed, for any $\varepsilon>0$ there exists $K$ such that $\alpha_{k} / \beta_{k}<\varepsilon$ for all $k \geq K$. Hence, for all $k \geq K$ we obtain

$$
\int_{\alpha_{k} / \beta_{k}}^{1} \xi^{-\frac{\delta\left(1-\varepsilon_{k}\right)}{\rho\left(\beta_{k}\right)\left(\rho\left(\alpha_{k}\right)-1\right)}} d \xi \geq \int_{\varepsilon}^{1} \xi^{-\frac{\delta\left(1-\varepsilon_{k}\right)}{\rho\left(\beta_{k}\right)\left(\rho\left(\alpha_{k}\right)-1\right)}} d \xi
$$


and thus,

$$
\liminf _{k \rightarrow \infty} \int_{\alpha_{k} / \beta_{k}}^{1} \xi^{-\frac{\delta\left(1-\varepsilon_{k}\right)}{\rho\left(\beta_{k}\right)\left(\rho\left(\alpha_{k}\right)-1\right)}} d \xi \geq \int_{\varepsilon}^{1} \xi^{-\frac{\delta}{q(q-1)}} d \xi \geq \log \frac{1}{\varepsilon}
$$

This proves (5.7), and therefore the proof is complete.

Remark 5.2. It is not difficult to show that the restrictions on $q$ and $\delta$ in Theorem 1.7 are sharp in the sense that $p_{q, \delta} \in A$ if $q(q-1)>\delta$.

\section{REFERENCES}

[1] R.R. Coifman and C. Fefferman, Weighted norm inequalities for maximal functions and singular integrals, Studia Math. 51 (1974), 241-250. MR0358205 (50:10670)

[2] D. Cruz-Uribe, L. Diening and A. Fiorenza, A new proof of the boundedness of maximal operators on variable Lebesgue spaces, submitted.

[3] D. Cruz-Uribe and A. Fiorenza, L $\log L$ results for the maximal operator in variable $L^{p}$ spaces, Trans. Amer. Math. Soc., 361 (2009), no. 5, 2631-2647. MR2471932

[4] D. Cruz-Uribe, A. Fiorenza and C. J. Neugebauer, The maximal function on variable $L^{p}$ spaces, Ann. Acad. Sci. Fenn. Math. 28 (2003), 223-238; corrections in Ann. Acad. Sci. Fenn. Math. 29 (2004), 247-249. MR.1976842 (2004c:42039); MR2041952 (2004m:42018)

[5] L. Diening, Maximal function on generalized Lebesgue spaces $L^{p(\cdot)}$, Math. Inequal. Appl. 7 (2004), no. 2, 245-253. MR2057643 (2005k:42048)

[6] L. Diening, Maximal functions on Musielak-Orlicz spaces and generalized Lebesgue spaces, Bull. Sci. Math. 129 (2005), no. 8, 657-700. MR2166733 (2006e:46032)

[7] L. Diening, P. Harjulehto, P. Hästö, Y. Mizuta and T. Shimomura, Maximal functions in variable exponent spaces: Limiting cases of the exponent, preprint.

[8] L. Diening, P. Hästö and A. Nekvinda, Open problems in variable exponent Lebesgue and Sobolev spaces, FSDONA 2004 Proceedings, pages 38-52, Academy of Sciences of the Czech Republic, Prague, 2005.

[9] B. Jawerth, Weighted inequalities for maximal operators: Linearization, localization and factorization, Amer. J. Math. 108 (1986), 361-414. MR833361 (87f:42048)

[10] E. Kapanadze and T. Kopaliani, A note on maximal operator on $L^{p(t)}(\Omega)$ spaces, Georgian Math. J., 15 (2008), no. 2, 307-316. MR 2428473 (2009d:42047)

[11] T.S. Kopaliani, Infimal convolution and Muckenhoupt $A_{p_{(\cdot)}}$ condition in variable $L^{p}$ spaces, Arch. Math. 89 (2007), no. 2, 185-192. MR2341730 (2008h:42021)

[12] O. Kováčik and J. Rákosník, On spaces $L^{p(x)}$ and $W^{k, p(x)}$, Czechoslovak Math. J. 41(116) (1991), no. 4, 592-618. MR1134951 (92m:46047)

[13] A.K. Lerner, Some remarks on the Hardy-Littlewood maximal function on variable $L^{p}$ spaces, Math. Z. 251 (2005), no. 3, 509-521. MR2190341 (2006g:42030)

[14] B. Muckenhoupt, Weighted norm inequalities for the Hardy maximal function, Trans. Amer. Math. Soc. 165 (1972), 207-226. MR0293384 (45:2461)

[15] E. Nakai and K. Yabuta, Pointwise multipliers for functions of bounded mean oscillation, J. Math. Soc. Japan 37 (1985), 207-218. MR780660 (87d:42020)

[16] A. Nekvinda, Hardy-Littlewood maximal operator on $L^{p(x)}\left(\mathbb{R}^{n}\right)$, Math. Inequal. Appl. 7 (2004), no. 2, 255-265. MR2057644 (2005f:42045)

[17] A. Nekvinda, A note on maximal operator on $\ell^{\left\{p_{n}\right\}}$ and $L^{p(x)}(\mathbb{R})$, J. Funct. Spaces Appl. 5 (2007), no. 1, 49-88. MR2296013 (2008c:46045)

[18] A. Nekvinda, Maximal operator on variable Lebesgue spaces for almost monotone radial exponent, J. Math. Anal. Appl. 337 (2008), no. 2, 1345-1365. MR2386383 (2009d:42038)

[19] L. Pick and M. Růžička, An example of a space of $L^{p(x)}$ on which the Hardy-Littlewood maximal operator is not bounded, Expo. Math. 19 (2001), no. 4, 369-371. MR.1876258 (2002m:42016)

[20] E.M. Stein, Singular Integrals and differentiability properties of functions, Princeton Univ. Press, 1970. MR0290095 (44:7280)

Department of Mathematics, Bar-Ilan University, Ramat-Gan 52900, Israel

E-mail address: aklerner@netvision.net.il 